Sir,

\section{Malignant melanoma and massive retinal gliosis in phthisis bulbi}

Eye (2002) 16, 781-782. doi:10.1038/sj.eye.6700217

Uveal melanoma is the commonest primary intraocular malignancy with an incidence of approximately 5-7 per million per year in the United States and Western Europe. ${ }^{1,2}$ The tumour may be asymptomatic, remaining undetected unless ophthalmoscopy is performed or it may give rise to symptoms due to its location or secondary retinal effects like exudative detachment. It has been long known that a phthisical eye may harbour an occult malignant melanoma, though very few such cases have been reported. Similarly, massive retinal gliosis is an uncommon finding in such eyes. We present a case in which a small uveal melanoma and extensive retinal gliosis were discovered in an eye enucleated for longstanding phthisis. This case re-emphasises the importance of subjecting all enucleated eyes to histopathological
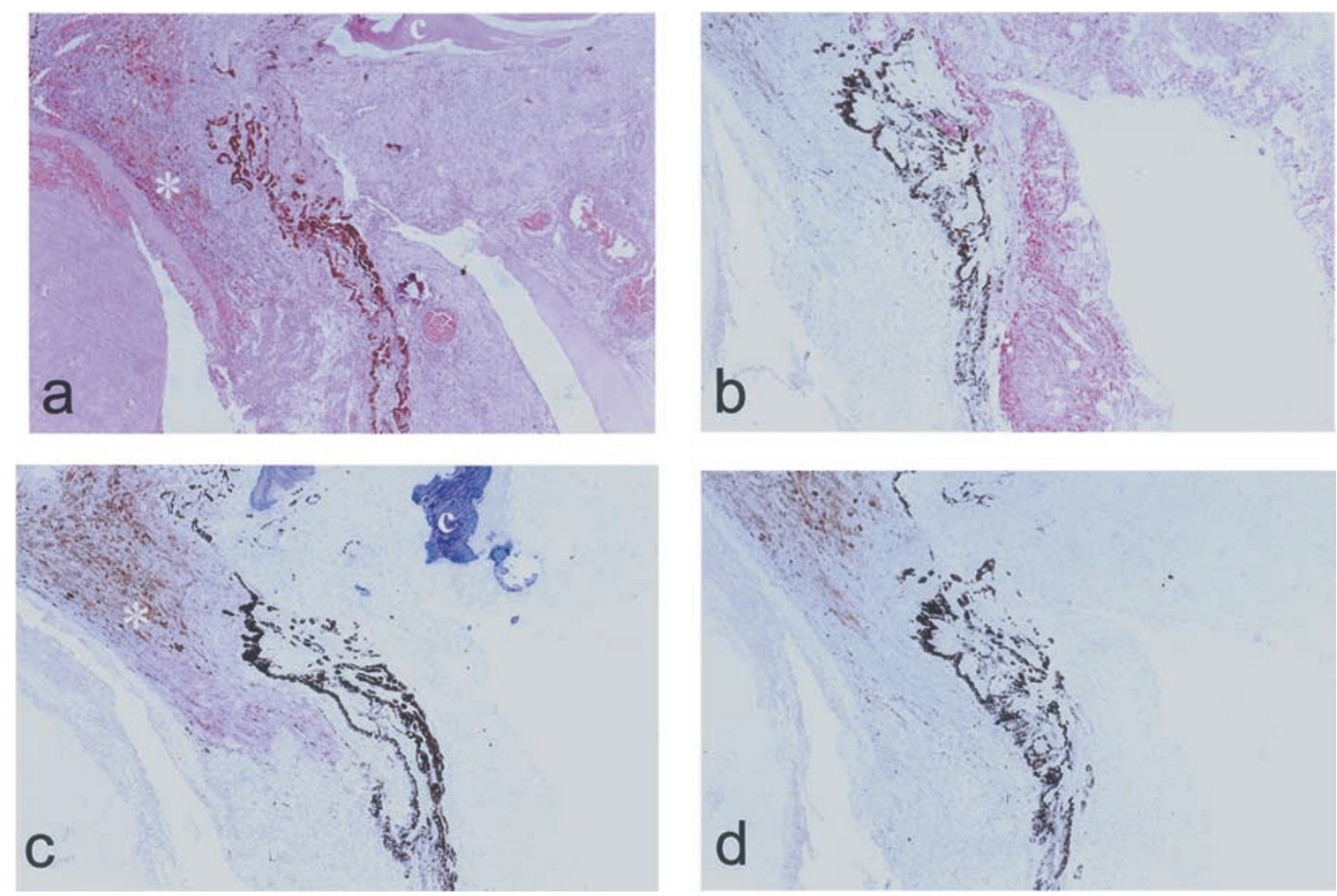

Figure 1 Histological sections through the malignant melanoma (asterisk) and part of retina in the enucleated eye: (a) stained with haematoxylin and eosin; (b)-(d) stained immunohistochemically for glial fibrillary acidic protein (GFAP-a glial marker), HMB45 (melanoma marker) and a control antibody respectively (red reaction product, haematoxylin counterstain). In (a), the malignant melanoma can be seen as a lightly pigmented plaque-like expansion of the anterior choroid beneath a disorganised, more heavily pigmented, pigment epithelium. The retina is also disorganised and grossly thickened, and contains engorged, partly hyalinised, blood vessels. In (b), the retina can be seen to be replaced by glial tissue. In (c), infiltrating HMB45-positive tumour cells extend anteriorly from the main plaque as well as around pigment epithelial clusters. Calcification (white c) is clearly seen in the adjacent area of massive retinal gliosis. (d) No reaction product is seen in the control section. $($ All $\times 40)$. 
glial in origin, indicating massive retinal gliosis. There was calcification in the retina with some osseous metaplasia at the RPE level and abnormal retinal blood vessels (Figure 1).

The anterior choroid contained a plaque-like proliferation of variably pigmented spindle cell melanocytes. Although the lesion was generally well demarcated, there was local infiltration of adjacent structures (Figure 1). The cells were immunoreactive for HMB45. The melanocytes showed anisonucleosis and occasional prominent nucleoli. These features were consistent with a spindle cell malignant melanoma. No evidence of extraocular extension was seen.

\section{Comments}

It has been long known that a phthisical eye may harbour an occult malignant melanoma, though very few such cases have been reported. Sarma et $a l^{3}$ reported a case of malignant melanoma in the left eye of a 62-year-old man who had been blind due to trauma for 35 years. Perry et al ${ }^{4}$ reported a case of occult choroidal malignant melanoma in an eye with spontaneous expulsive choroidal haemorrhage and suggested that this association may be more than a chance occurrence, inasmuch as both are associated with necrosis of the posterior ciliary arteries.

Undiagnosed malignant melanoma of choroid is uncommon in phthisical eyes. Massive retinal gliosis is also an uncommon finding seen in eyes enucleated for phthisis bulbi. The case reported here shows both these rare features. It has been reported earlier that retinal gliosis may simulate a choroidal malignant melanoma ${ }^{5,6}$ and also that ultrasound reflectivity and magnetic resonance imaging findings of massive retinal gliosis may resemble those of choroidal malignant melanoma. However, to the best of the authors' knowledge, the presented case is the first one to exhibit histological evidence of both malignant melanoma of choroid and massive retinal gliosis. It is possible that factors that lead to massive retinal gliosis in this phthisical eye, also lead to atypical proliferation of melanocytes giving rise to malignant melanoma. One may speculate that necrosis of posterior ciliary arteries was a precipitating factor for suprachoroidal haemorrhage, retinal gliosis and malignant melanoma formation in our case. Whatever the cause, this case once again emphasises the importance of subjecting enucleated eyes to a histopathological examination since the discovery of a malignant melanoma would influence the clinical management of such patients.

\section{References}

1 Scotto J, Fraumeni JF Jr, Lee JA. Melanomas of the eye and other noncutaneous sites: epidemiologic aspects. $\mathrm{JNCI}$ 1976; 56: 489-491.

2 Keller AZ. Histology, survivorship, and related factors in the epidemiology of eye cancers. Am J Epidemiol 1973; 97: 386-393.

3 Sarma DP, Deshotels SJ Jr, Lunseth JH. Malignant melanoma in a blind eye. J Surg Oncol 1983; 23: 169-172.

4 Perry HD, Hsieh RC, Evans RM. Malignant melanoma of the choroids associated with spontaneous expulsive choroidal haemorrhage. Am I Ophthalmol 1977; 84: 205 208

5 Berger B, Peyman GA, Juarez C, Mason G, Raichand M. Massive retinal gliosis simulating choroidal melanoma. Can J Ophthalmol 1979; 14: 285-290.

6 Sahel JA, Frederick AR Jr, Pesavento R, Albert DM. Idiopathic retinal gliosis mimicking a choroidal melanoma. Retina 1988; 8: 282-287.

A Tripathi, P Hiscott and BE Damato

St Paul's Eye Unit

Ocular Oncology Services

Royal Liverpool University Hospital

Liverpool, UK

Correspondence: A Tripathi

68 Kingsleigh Road

Stockport, Cheshire

SK4 3PP, UK

Tel: (0161) 4326310

Fax: (7092) 285960

E-mail: ajtrip@hotmail.com

Sir,

Cataract surgery in Senior-Loken syndrome is beneficial despite severe retinopathy Eye (2002) 16, 782-785. doi:10.1038/sj.eye.6700171

A common problem in patients with retinopathy is whether they will benefit from surgical correction of co-existent cataracts. ${ }^{1}$ The timing of surgery is dependent upon a clinical assessment of the degree of cataract compared to severity of retinopathy. The case presented here illustrates such a dilemma where cataract surgery exceeded all expectations of predicted benefit in the context of severe, widespread retinopathy.

\section{Case report}

A 28-year-old female was referred to our ophthalmic department in 1995. Her optometrist had noted reduced acuity and cataracts. Other medical history of 